\title{
IWPAAMS2007-03: Multiagent System For Predicting The Co2 Exchange In The North Atlantic Ocean
}

\author{
Javier Bajo, Vidal Alonso y Juan M. Corchado
}

\begin{abstract}
This paper presents a multiagent system developed to predict the behaviour of the Atlantic Ocean in relation to the sinks/sources of $\mathrm{CO}$. The heart of the multiagent system is an intelligent agent capable of automatically making predictions about the flux of $\mathrm{CO} 2$ in the North Atlantic ocean. The multiagent system has been tested in simulation conditions and this work presents the preliminary obtained results.
\end{abstract} $\mathrm{CO2}$.

Keywords- Multiagent system, CBR-BDI, Neural networks,

\section{INTRODUCCIÓN}

$\mathrm{L}$ os sistemas multiagente (SMA) son aplicaciones informáticas distribuidas con autonomía y cierto grado de "inteligencia", cuyos componentes son agentes. Un agente es una entidad que posee ciertas características, tales como autonomía, situación, reactividad, proactividad, habilidad social, aprendizaje, movilidad u organización [[20]]. La mayor parte de las arquitecturas actuales se basan en el modelo BDI [[4]]. Sin embargo, una de las principales carencias del modelo BDI se encuentra en que se carece de mecanismos eficientes de gestión de la memoria y de las experiencias pasadas. Una de las posibilidades para mejorar la eficiencia de la arquitectura BDI es la utilizar sistemas de razonamiento basado en casos (CBR) [[1]], como mecanismo de razonamiento. De esta forma se consiguen agentes CBR-BDI [[5]], con una gran capacidad de aprendizaje y de adaptación. Hoy en día es un hecho la creciente utilización de los agentes y sistemas multiagente para el desarrollo de aplicaciones en entornos dinámicos y flexibles tales como la Web, sistemas de control, robótica, etc. En este documento se presenta una arquitectura distribuida cuya principal característica es la

Este trabajo se ha llevado a cabo gracias a los proyectos MCYT TIC200307369-CO2-02 y CAXIS de PML.

Javier Bajo, Escuela Universitaria de Informática de la Universidad Pontificia de Salamanca, C/Compañía 5, 37002, Salamanca, España (correo electrónico: jbajope@upsa.es).

Juan M. Corchado, Departamento de Informática y Automática de la Universidad de Salamanca, Plaza de la Merced s/n, 37008, Salamanca, España (correo electrónico: corchado@usal.es).

Vidal Alonso, Escuela Universitaria de Informática de la Universidad Pontificia de Salamanca, C/Compañía 5, 37002, Salamanca, España (correo electrónico: valonsose@upsa.es). utilización de agentes CBR-BDI [[7]] para la monitorización y la predicción del intercambio de $\mathrm{CO}_{2}$ existente entre la atmósfera y la superficie de las aguas oceánicas, basado en la utilización de los datos obtenidos a través de técnicas de teledetección. Las características del problema de la interacción mar-aire hacen que resulte muy adecuada la utilización de un SMA para la generación de modelos dinámicos de forma automática.

Se denomina agente CBR-BDI [[5]] a un agente deliberativo BDI que incorpora un sistema CBR como motor de razonamiento. Estos agentes son capaces de aprender, a partir de un conocimiento inicial, interaccionar autónomamente con el entorno y con los usuarios del sistema y se adaptan a las necesidades del entorno. En el modelo BDI la estructura interna de un agente y su capacidad de elección se basan en aptitudes mentales. Esto tiene la ventaja de utilizan un modelo natural (humano) y de alto nivel de abstracción. El modelo BDI utiliza Beliefs (Creencias) como aptitudes informacionales, Desires (Deseos) como aptitudes motivacionales e Intentions (Intenciones) como aptitudes deliberativas de los agentes. El método propuesto en [[5], [10]] facilita la incorporación de sistemas CBR como mecanismo deliberativo en agentes $\mathrm{BDI}$, proporcionando un aprendizaje, adaptación y un mayor grado de autonomía que una arquitectura BDI pura [[4]]. El CBR es un paradigma que se basa en la idea de que los problemas similares tienen soluciones similares. De esta forma, un problema nuevo se resuelve consultando en la memoria experiencias similares que se hubiesen resuelto en el pasado.

En la siguiente sección se presenta el problema oceánico/atmosférico que motiva la mayor parte de esta investigación. En la tercera sección se muestra el sistema multiagente desarrollado. En la sección 4 se analiza la estructura interna del agente CBR-BDI encargado de generar modelos y realizar predicciones. Finalmente se presentan unos resultados preliminares y las conclusiones obtenidas.

\section{Problema de la Interacción Mar-Aire}

Uno de los factores más preocupantes que influyen en el comportamiento climatológico es la cantidad de $\mathrm{CO}_{2}$ presente en la atmósfera. El $\mathrm{CO}_{2}$ es uno de los gases de efecto invernadero, que contribuye a que la Tierra tenga una temperatura habitable, siempre y cuando se mantenga en unas cantidades determinadas [[16]]. Tradicionalmente se ha 
considerado que el principal sistema regulador de la cantidad de $\mathrm{CO}_{2}$ en la atmósfera es la fotosíntesis y respiración realizado por las plantas. Sin embargo, y especialmente gracias a la utilización de técnicas de teledetección se ha descubierto que el papel que juega el océano en la regulación de las cantidades de carbono es muy importante y es una magnitud que permanece indefinida [[17]]. La tecnología actual permite obtener datos y realizar cálculos impensables hasta hace poco tiempo. Estos datos proporcionan conocimiento sobre las fuentes de origen y disminución de $\mathrm{CO}_{2}$ así como de sus causas [[13]], lo que supone la posibilidad de realizar predicciones sobre el comportamiento futuro del $\mathrm{CO}_{2}$.

El sistema multiagente que se presenta tiene como objetivo modelar el flujo de $\mathrm{CO}_{2}$ intercambiado entre la atmósfera y las aguas de la superficie oceánica. Los principales parámetros a tener en cuenta a la hora de modelar el intercambio de $\mathrm{CO}_{2}$ entre la atmósfera y la superficie oceánica son: la temperatura del agua y del aire, la salinidad del agua, las presiones atmosféricas e hidrostáticas, la presencia de nutrientes y el vector velocidad del viento (en módulo y dirección). Estos parámetros pueden obtenerse a través de mediciones in-situ realizadas desde barcos oceanográficos o bien a través de imágenes de satélite. La información de satélite es vital para la construcción de modelos oceanográficos, y en este caso, para generar estimaciones de los flujos de $\mathrm{CO}_{2}$ entre el aire y el mar con una alta resolución tanto espacial como temporal, utilizando modelos de inteligencia artificial que pueden ser contrastados con la realidad por medio de muestreos directos in situ sobre el $\mathrm{CO}_{2}$ de la superficie del océano. Será necesario utilizar sistemas distribuidos automatizados y capaces de incorporar nuevo conocimiento para poder manejar todos los datos útiles y para crear modelos diarios en un tiempo y coste razonables. Nuestra propuesta se presenta en la siguiente sección.

\section{SistemA MUlTiAgENTE PARA LA INTERACCiÓN MAR-AIRE}

El objetivo final de este trabajo es construir un gran sistema multiagente organizado en subsistemas, de tal forma que cada subsistema se encargue de modelar el intercambio de $\mathrm{CO}_{2}$ en una zona del océano con unas determinadas características. Cada subsistema se comunicará con el resto de los subsistemas, obteniendo un intercambio de información que ayude a la creación de modelos y que facilite las predicciones. Dado que se trata de un proyecto de gran magnitud, en este trabajo se presenta el estudio y desarrollo de un subsistema, cuya arquitectura final puede observarse en la Figura 1.

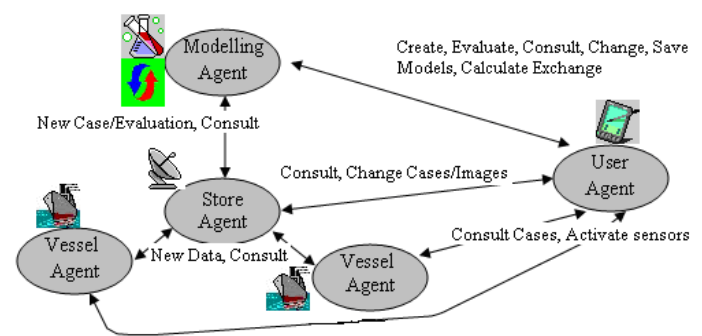

Fig. 1. SMA correspondiente a un subsistema para una zona concreta del Océano.
Por el momento se ha desarrollado el prototipo correspondiente a un subsistema como el mostrado en la Figura 1, en la que es posible observar como en un subsistema participan cuatro tipos diferentes de agentes: un tipo de agente Modelling, encargado de la creación y evaluación de modelos en función de los datos recibidos desde los agentes Store, Vessel o User, así como de la obtención de predicciones; un tipo de agente Store, encargado de almacenar los datos obtenidos a partir de imágenes de satélite; un tipo de agente Vessel, encargado de gestionar los datos obtenidos por medio de un barco ocenanográfico; y un tipo agente User que permite a los oceanógrafos interactuar con el sistema multiagente.

Los modelos generados por el agente Modelling permiten predecir el intercambio de $\mathrm{CO}_{2}$ entre la superficie del océano y la atmósfera. Para crear un nuevo modelo es necesario disponer de información proporcione una descripción inicial del problema a modelar. La descripción del problema se obtiene por medio de los datos obtenidos a través de imágenes de satélite. El agente Store se encarga de procesar las imágenes de satélite y transformarlas para que el sistema pueda utilizarlas. En la Figura 2 es posible apreciar una captura de pantalla correspondiente al agente Store. En ella se muestran los datos obtenidos por medio de imágenes de satélite en un rango de fechas, para un determinado intervalo de latitudes y longitudes. Los datos que se obtienen a partir del procesamiento de imágenes de satélite son principalmente datos de clorofila, salinidad y temperatura. Para transformar las imágenes de satélite el agente Store utiliza algoritmos [[8], [12]]. La Figura 2 muestra los datos ya procesados obtenidos para zonas concretas del Atlántico Norte. Los datos obtenidos son enviados al agente Modelling para que pueda crear nuevos modelos. Estos modelos tienen que ser evaluados para comprar su bondad. Para ello se utiliza la información obtenida por medio de cada agente Vessel que se instala en un navío y recoge datos in-situ que pueden permiten contrastar los resultados obtenidos por medio de los modelos creados por el agente Modelling. El agente usuario puede interactuar con cualquier agente del sistema y puede ser ejecutado sobre dispositivos móviles, tales como PDAs, lo cual facilita enormemente el trabajo de los oceanógrafos, permitiendo una gran libertad de movimientos e independencia de la localización. Cada oceanógrafo dispone de un agente de usuario situado en un dispositivo ligero que le permite acceder al SMA de forma inalámbrica.

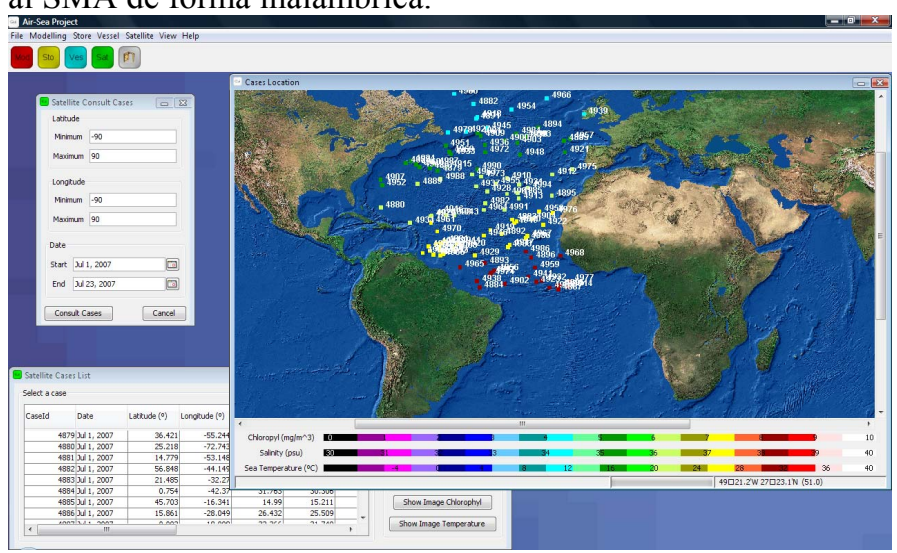

Fig. 2. Agente Store. Casos obtenidos a partir de imágenes de satélite. 
La implementación del sistema se ha realizado utilizando la plataforma Jade [[3]] y la herramienta Jadex [[15]], que es una extensión que permite dotar de una arquitectura BDI a los agentes Jade. De esta forma los agentes Jadex trabajan con conceptos de beliefs (creencias), goals (metas) y plans (planes). Jadex tiene la ventaja de permitir que el programador pueda introducir sus propios mecanismos deliberativos. En nuestro caso este mecanismo va a ser un sistema CBR. El agente Modelling es un agente Jadex, mientras que el resto de agentes se construyen con Jade. Además, una de las ventajas de utilizar Jadex radica en que es posible utilizar los mecanismos de comunicación utilizados por Jade [[3], [15]].

\section{Agente PARA la CREAción de Modelos OCEANOGRÁFICOS}

El componente principal de la arquitectura propuesta es el agente Modelling, encargado de generar modelos y realizar predicciones. Se trata de un agente deliberativo BDI [[20]] que utiliza un sistema CBR como motor de razonamiento. Un sistema CBR maneja casos. Un caso es una experiencia pasada, que incluye una descripción del problema, la solución utilizada para resolverlo y el resultado obtenido. Construir un agente CBR-BDI [[5], [10]] supone por un lado integrar el concepto de caso en la estructura del modelo BDI y por otro lado que el agente BDI ejecute el ciclo CBR. De esta forma la generación de un modelo supone la ejecución de un ciclo de razonamiento $\mathrm{CBR}$ formado por cuatro etapas secuenciales: retrieval, reuse, revise y retain. La etapa retrieval consiste en recuperar de la memoria de casos aquellos casos que poseean una descripción de problema similar al problema actual. La etapa reuse consiste en adaptar las soluciones correspondientes a las descripciones de problema similares recuperadas para crear una nueva solución. En la etapa revise se evalúa la solución proporcionada. Por último, en la etapa retain, se aprende de la nueva experiencia. Tal y como se puede apreciar en la Figura 3, el agente CBR-BDI utiliza sus estructuras internas para representar un caso: creencias, deseos e intenciones. Creencias para representar la descripción del problema, una creencia de tipo ProblemDescription (obteniendo una descripción de problema como la mostrada en la Tabla 1: fecha, latitud, longitud, salinidad, clorofila, viento, presiones parciales y temperaturas). Deseos para representar las metas que el agente persigue (predecir el flujo de $\mathrm{CO}_{2}$ intercambiado, calcular los mejores parámetros de predicción para distintos tamaños de ventana, y calcular la mejor ventana de predicción para un determinado \% de error máximo permitido). Intenciones o secuencias de acciones para alcanzar una o varias metas en función de las creencias de las que se dispone.

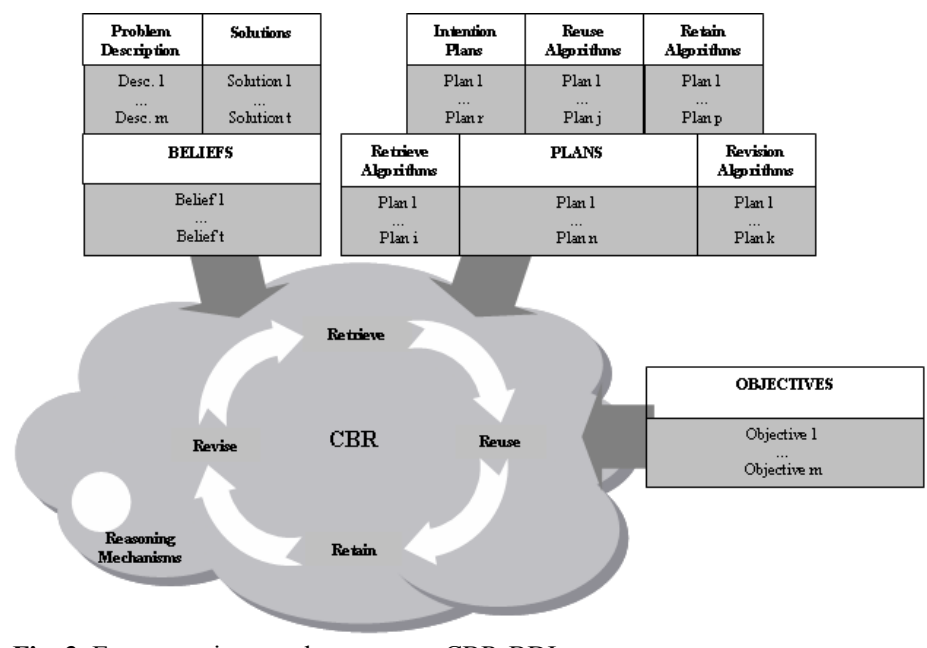

Fig. 3. Estructura interna de un agente CBR-BDI.

TABLA I

Atributos DE LOS CASOS

\begin{tabular}{ll}
\hline $\begin{array}{l}\text { Atributo del } \\
\text { Caso }\end{array}$ & Valor \\
\hline DATE & Fecha (dd/mm/yyyy) \\
\hline LAT & Latitud (grados decimales) \\
\hline LONG & Longitud (grados decimales) \\
\hline SST & Temperatura $\left({ }^{\circ} \mathrm{C}\right)$ \\
\hline S & Salinidad (sin unidades) \\
\hline WS & Fuerza del viento $(\mathrm{m} / \mathrm{s})$ \\
\hline WD & Dirección del viento $($ sin unidades) \\
\hline Fluo_calibrated & Fluorescencia calibrada con clofofila \\
\hline SW pCO $\mathrm{CO}_{2}$ & $\begin{array}{l}\text { Presión parcial de } \mathrm{CO}_{2} \text { en la superficie marina } \\
\text { (micro Atmósferas) }\end{array}$ \\
\hline Air $\mathrm{pCO}_{2}$ & $\begin{array}{l}\text { Presión parcial de } \mathrm{CO}_{2} \text { en el aire }(\mathrm{micro} \\
\text { Atmósferas) }\end{array}$ \\
\hline Flux of $\mathrm{CO}_{2}$ & Flujo de $\mathrm{CO}_{2}$ intercambiado $\left(\right.$ Moles $\left./ \mathrm{m}^{2}\right)$ \\
\hline
\end{tabular}

La forma de implantar el ciclo CBR dentro de un agente BDI consiste en que el agente BDI ejecute las etapas del ciclo CBR como capacidades [[1]]. Un agente BDI posee capacidades (funcionalidades que el agente es capaz de realizar por sí mismo sin necesidad de contactar con otros agentes) y ofrece servicios a otros agentes. Como se puede observar en la Figura 4, el agente Modelling ofrece cuatro servicios (Obtain Exchange, Obtain Model, Obtain Evaluation y Construct Model) y posee seis capacidades (Forecast Exchange Rate, Evaluate Model, Consult Model, Jacobean Sensivity Matriz - JSM, Pondered Weigh Technique - PWT y Revision Simulated Equation - RSE). 


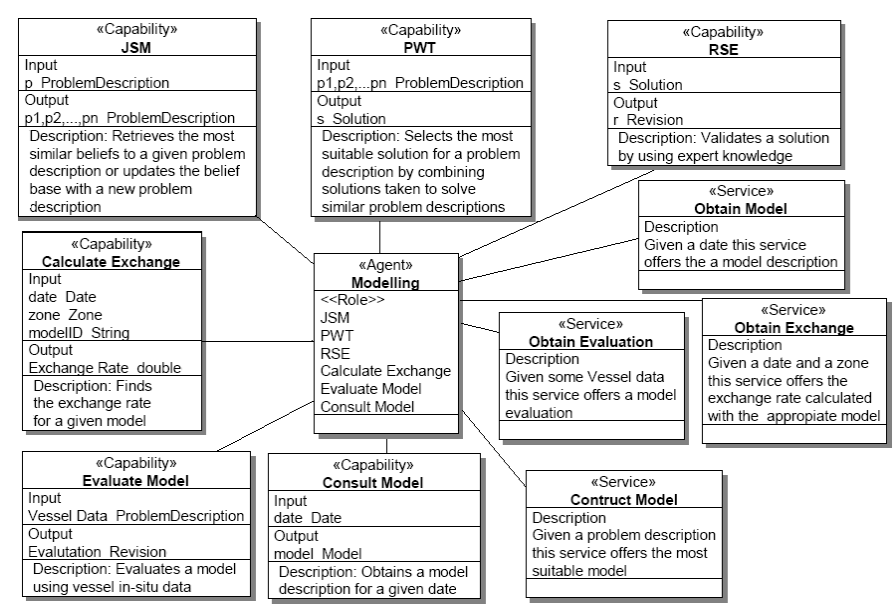

Fig. 4. Diagrama de clases para el agente Modelling.

Las tres últimas capacidades constituyen el ciclo CBR, y su funcionamiento cuando se recibe una nueva descripción de problema es el que a continuación pasa a describirse: En la etapa de recuperación la capacidad JSM permite obtener aquellos casos con una descripción de problema más similar a la del caso actual [[14]]. Para ello se utiliza una red neuronal que permite obtener las componentes principales de la memoria de casos, y conocer cuales de las variables de entrada (que forman la descripción del problema) tienen mayor influencia a la hora de crear clusters. Si consideramos JSM una matriz NxM, el elemento $S_{k i}$ en la matriz representa la sensibilidad (influencia) de la salida $k$ sobre la entrada $i$, como muestra la ecuación 1.

$$
S_{k i}=\frac{\partial y_{k}}{\partial x_{i}}=\frac{\partial f_{k}\left(\text { net }_{k}\right)}{\partial x_{i}}=\frac{\partial f_{k}\left(\text { net }_{k}\right)}{\partial n e t_{k}} \frac{\partial n e t_{k}}{\partial y_{j}} \frac{\partial y_{j}}{\partial n e t_{j}} \frac{\partial \text { net }_{j}}{\partial x_{i}}=\frac{\partial f_{k}\left(\text { net }_{k}\right)}{\partial n e t_{k}}\left(\sum_{j=1}^{H} w_{k j} \frac{\partial f_{j}\left(\text { net }_{j}\right)}{\partial n e t_{j}} w_{j i}\right)
$$

Donde $w_{i j}$ es el peso de la conexión entre la neurona de entrada $i$ y la neurona oculta $j . w_{k j}$ es el peso de la conexión entre la neurona oculta $\mathrm{j}$ y la neurona de salida k. $y_{k}$ es la salida de la neurona $\mathrm{k}$ de la capa de salida $y_{k}=f_{k}\left(\right.$ net $\left._{k}\right)$, y $y_{j}$ es la salida de la neurona $j$ de la capa oculta $y_{j}=f_{j}\left(n e t_{j}\right) . f_{j}$ es la función de activación de la neurona $h$ y net $t_{j}$, net $t_{k}$ se obtienen a partir de las ecuaciones 2 y 3 respectivamente. Siendo $H$ el número de neuronas de la capa oculta, y $\theta_{j}$ y $\theta_{k}$ los umbrales permitidos. Una vez obtenidas las componentes principales se busca en la memoria de casos aquellos con componentes principales más similares a la descripción del problema actual. Para ello se utiliza un algoritmo de similitud basado en la función coseno.

$$
\begin{aligned}
& n^{n e t_{j}}=\sum_{i=1}^{N} w_{j i} x_{i}+\theta_{j} \\
& n e t_{k}=\sum_{j=1}^{H} w_{k j} y_{j}+\theta_{k}
\end{aligned}
$$

En la etapa de adaptación se combinan las soluciones de los casos similares recuperados para obtener una solución adecuada al problema actual. Se efectúa mediante la capacidad PWT, en la que los casos se ponderan [[9]] y se asigna el mayor peso al caso con una descripción de problema más similar a la del caso actual.

$$
p^{*}=\frac{1}{\sum_{r=1}^{Z} e^{-|a-r|}} \sum_{r=1}^{Z} e^{-|a-r|} p^{r}
$$

La ecuación 4 muestra el proceso de adaptación, donde $p^{*}$ es la predicción, $Z$ el número de casos similares y $p^{r}$ son las predicciones (soluciones) correspondientes a cada caso pasado, $a$ es la medida de mínima similitud para los casos recuperados y $r$ es la medida de similitud para el caso r-ésimo. Se utiliza una red neuronal que se entrena con los casos similares recuperados y sus respectivas soluciones. Una vez entrenada se le proporciona el caso actual y se obtiene su solución.

En la etapa de revisión cada modelo se revisa en la capacidad RSE. Para ello se utiliza la ecuación 5 con los coeficientes de intercambio de Wanninkhof [[18]].

$$
F=k s o\left(p \mathrm{CO}_{2} \mathrm{SW}-p \mathrm{CO}_{2} \mathrm{AIR}\right)
$$

Finalmente, en la etapa de aprendizaje se compara el valor F con el valor de la predicción. Si la diferencia es inferior al $10 \%$, el caso se almacena en la base de creencias. La etapa de aprendizaje también se efectúa en la capacidad RSE. En este caso se ha considerado que el aprendizaje resulta más eficiente si se tienen en cuenta las experiencias positivas, desechado las negativas. La razón está en la gran cantidad de datos disponibles.

\section{Resultados y CONClusiones}

El sistema descrito ha sido probado con datos recogidos en el Océaho Atlántico Norte durante 2004. A pesar de que el sistema no se encuentra totalmente operativo y que el objetivo del proyecto es el de construir un prototipo para investigación y no una herramienta comercial, los resultados iniciales han sido muy satisfactorios desde los puntos de vista técnico y científico. La construcción del sistema distribuido ha sido relativamente fácil gracias a la utilización de bibliotecas CBRBDI previamente desarrolladas [[1], [2], [5], [6]]. El formalismo definido en [[10]] facilita un paso directo entre la definición de agente y la construcción CBR. La utilización de dispositivos inalámbricos ha sido valorada positivamente por los oceanógrafos, indicando que proporciona una gran movilidad y facilidad de acceso al sistema. Los interfaces se han adaptado para poder ser ejecutados tanto en ordenadores personales como en dispositivos móviles, caracterizados por su limitación en las capacidades de almacenamiento y procesamiento. La Figura 5 muestra dos capturas de pantalla correspondientes al interfaz de un agente Vessel. En la imagen de la izquierda es posible apreciar la misión realizada por el barco y los datos obtenidos. En la imagen de la derecha se muestra la ruta seguida por el barco, utilizando la herramienta Google Earth [[11]], que ha sido integrada con el sistema. 

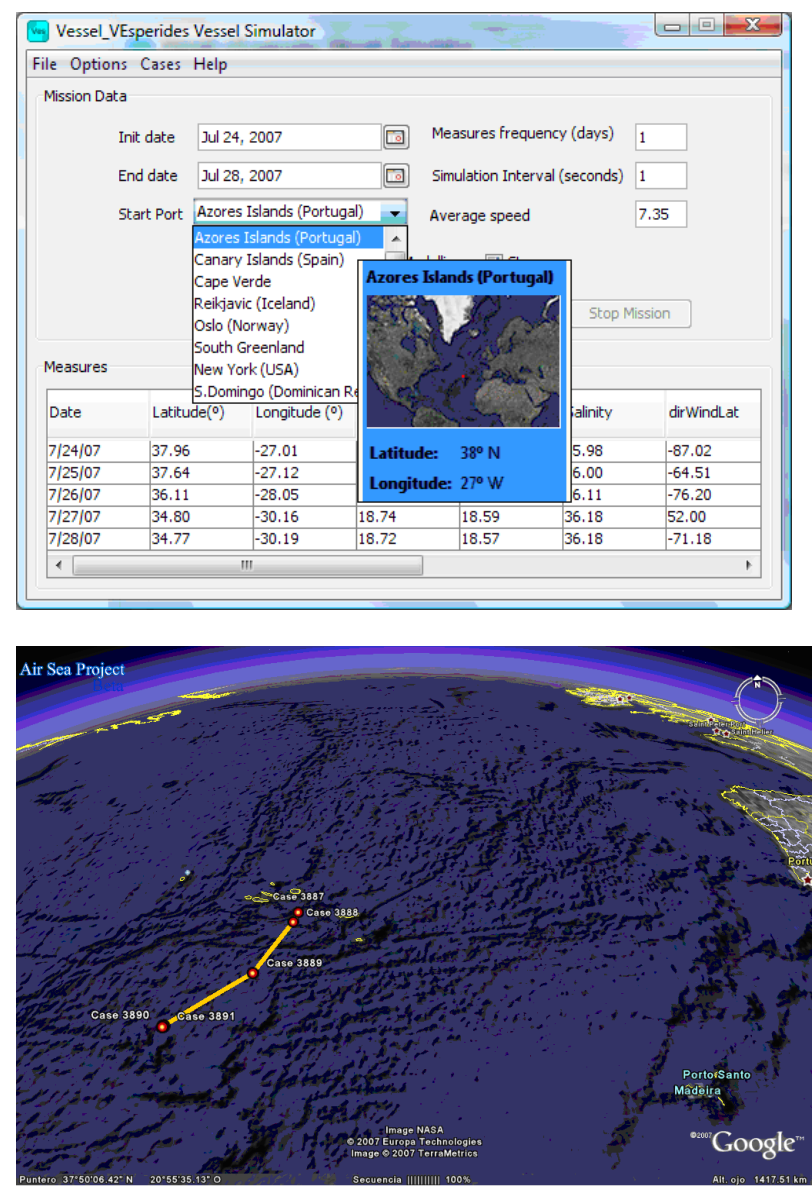

Fig. 5. Utilización de la herramienta Google

La Figura 6 muestra el interfaz correspondiente a un agente Modelling, concretamente para la generación de un modelo. En la Figura 6 es posible observar cómo un oceanógrafo puede configurar manualmente cada uno de los algoritmos utilizados en cada etapa del ciclo CBR. La interacción con el SMA por parte de desarrolladores y oceanógrafos ha sido continua durante su construcción y periodo de prueba. El sistema ha sido probado bajo condiciones de simulación de tal forma que los agentes Store y Vessel se han construido incluyendo simuladores que generan casos a partir de los datos reales obtenidos en el Océano Atlántico $( \pm 37 \mathrm{~N}, 25 \mathrm{~W})$. Bajo estas condiciones los modelos propuestos por el sistema multiagente han sido cada vez más precisos. Sin embargo, si el número de casos manejado es muy elevado la eficiencia del sistema disminuye. La Figura 7 muestra una comparativa entre los datos reales y las predicciones realizadas por el SMA trabajando con datos relativos a meses de 2003-2004.

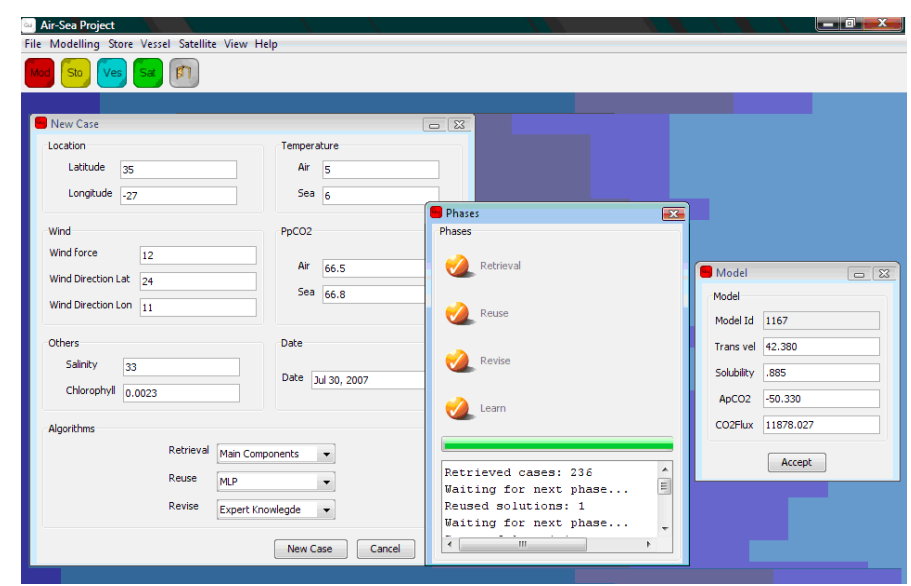

Fig. 6. Captura de pantalla de un agente Modelling correspondiente a la creación de un modelo.

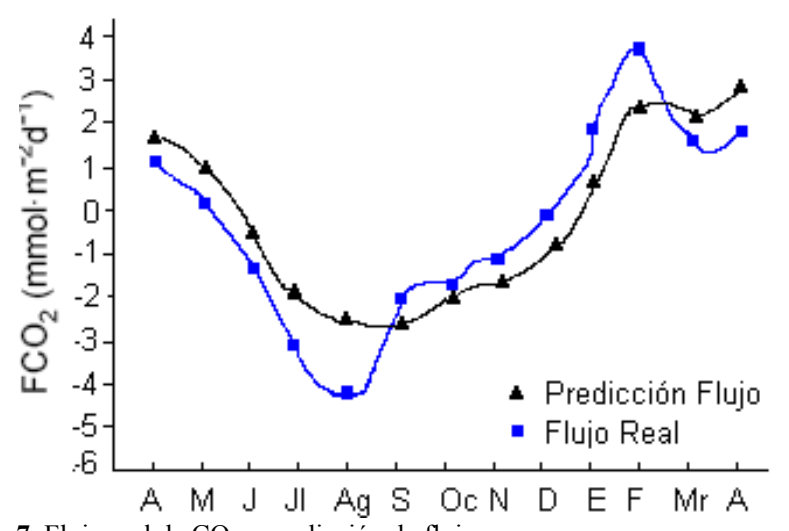

Fig. 7. Flujo real de $\mathrm{CO}_{2}$ y predicción de flujo.

Como puede observarse en la Figura 7 las predicciones realizadas por el SMA son bastante precisas (9 de los 12 modelos fueron aceptados como válidos). El SMA realiza predicciones basándose en su experiencia y en la similitud con situaciones pasadas. Claramente la precisión de las predicciones mejora a medida que el número de casos disponibles aumenta. Por otro lado es necesario controlar que el número de casos manejado no sea excesivo. Para ello se ha utilizado un sistema de prioridades basado en crear una estructura piramidal de eficiencias que permite clasificar los casos en base a la eficiencia obtenida. El sistema facilita la incorporación de nuevos agentes que utilicen diferentes técnicas de modelado y estrategias de aprendizaje de forma que se puedan realizar experimentos adicionales con nuevas técnicas y puedan ser comparados con los resultados iniciales presentados en este documento.

\section{REFERENCIAS}

[1] A. and Plaza E.: Case-Based Reasoning: foundational Issues, Methodological Variations, and System Approaches, AICOM. Vol. 7., pp 39-59 (1994)

[2] Bajo J. and Corchado J.M (2005). Evaluation and monitoring of the airsea interaction using a CBR-Agents approach. LNAI 3620, pp. 50-62. Springer Verlag

[3] Bajo J. and Corchado J.M. (2006). Multiagent architecture for monitoring the North-Atlantic carbon dioxide Exchange rate. CAEPIA 2005. LNCS 4177, pp 321-330

[4] Bellifime, F. Poggi, A. and Rimasa, G. (2001) JADE: a FIPA2000 compliant agent development environement. Agents 2001 pp. 216-217. 
[5] Bratman, M.E. (1987). Intentions, Plans and Practical Reason. Harvard University Press, Cambridge, M.A.

[6] Corchado J. M. and Laza R. (2003). Constructing Deliberative Agents with Case-based Reasoning Technology, International Journal of Intelligent Systems. Vol 18, No. 12, December. pp.: 1227-1241

[7] Corchado J.M., Bajo J., de Paz Y. y Tapia D. I.: Intelligent Environment for Monitoring Alzheimer Patients, Agent Technology for Health Care. Decision Support Systems. Eslevier Science. In Press (2007)

[8] Corchado J.M., Glez-Bedia M., de Paz Y., Bajo J. y de Paz J.F. Concept, formulation and mechanism for agent replanification: MRP Architecture. Computational Intelligence. Blackwell Publishers. In Press (2008)

[9] Dransfeld S., Tatnall A.R., Robinson I. S. and Mobley C.D. (2005). Prioritizing ocean colour channels by neural network input reflectance perturbation. International Journal of Remote Sensing, 26, (5), 0431048.

[10] De Paz Santana Y (2005) Mixture Weibull distribution using artificial neural networks with csnsurated data PHD thesis, chapter 3 .

[11] Glez-Bedia M., Corchado J. M., Corchado E. S. and Fyfe C. (2002) Analytical Model for Constructing Deliberative Agents, Engineering Intelligent Systems, Vol 3: pp. 173-185.

[12] Google Earth (2007) www.google.com.

[13] Lavencer S.J., Pinkerton M.H., Froidefond J.M., Morales J., Aiken J. and Moore J.F. (2004) SeaWiFS validation in European coastal waters using optical and bio-geochemical measurements. International Journal of Remote Sensing, Vol. 25, No. 7-8, pp. 1481-1488

[14] Lefevre N., Aiken J., Rutllant J., Daneri G., Lavender S. and Smyth T. (2002) Observations of pCO2 in the coastal upwelling off Chile: Sapatial and temporal extrapolation using satellite data. Journal of Geophysical research. Vol. 107, no. 0

[15] Montaño Moreno J.J. and Palmer Pol A. (2002). Artificial Neural Networks, opening the black box. Metodología de las Ciencias del Comportamiento 4(1) 77-93.

[16] Pokahr, A., Braubach, L. and Lamersdorf W. (2003) Jadex: Implementing a BDI-Infrastructure for JADE Agents, in: EXP - In Search of Innovation (Special Issue on JADE), Vol 3, Nr. 3, Telecom Italia Lab, Turin, Italy, September 2003, pp. 76-85.

[17] Sarmiento J. L. and Dender M. (1994) Carbon biogeochemistry and climate change. Photosynthesis Research, Vol. 39, 209-234.

[18] Takahashi T., Olafsson J., Goddard J. G., Chipman D. W. and Sutherland S. C. (1993) Seasonal Variation of CO2 and nutrients in the High-latitude surface oceans: a comparative study. Global biochemical Cycles. Vol. 7, no. 4. pp 843-878.

[19] Wanninkhof, R. (1992). Relationship between wind speed and gas exchange over the ocean. J. Geophys. Res. 97, 7, 373-7, 383.

[20] Wooldridge M. and Jennings N.R. (1995) Agent Theories, Architectures, and Languages: a Survey. Wooldridge and Jennings, editors, Intelligent Agents, Springer-Verlag, pp. 1-22.

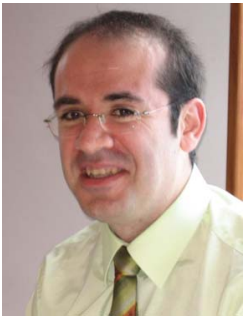

Javier Bajo Actualmente es Profesor Contratado Adjunto en la Universidad Pontificia de Salamanca (España). Obtuvo un doctorado en Ciencias de la Computación e Inteligencia Artificial y un Master en Comercio Electrónico por la Universidad de Salamanca en 2007. Finalizó sus estudios de Ingeniero Técnico en Informática de Sistemas por la Universidad de Valladolid (España) en 2001 y sus estudios de Ingeniero en Informático en la Universidad Pontificia de Salamanca (España) en 2003. Ha sido miembro de comités de organización y comités científicos en números congresos internacionales como IWPAAMS, CAEPIA, IDEAL, HAIS, etc. y co-autor de más de 80 artículos en revistas internacionales de reconocido prestigio, libros, capítulos de libros, workshops y simposios.

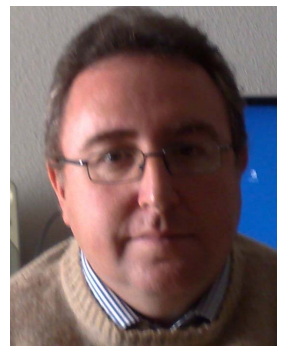

Vidal Alonso Actualmente es Profesor Numerario en la Universidad Pontificia de Salamanca (España). Obtuvo un doctorado en Informática por la Universidad Pontificia de Salamanca (España) en 2004. Es Director de la Escuela Universitaria de Informática de Universidad Pontificia de Salamanca desde 1999, habiendo sido anteriormente Sub-director (1998-1999), Jefe de Estudios (1998-1999) y Secretario (1996-1998). Ha sido miembro de comités de organización y comités científicos en números congresos internacionales y co-autor de artículos publicados en revistas internacionales de reconocido prestigio, workshops y simposios.

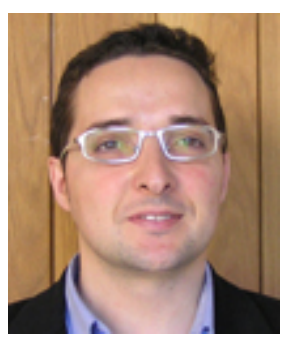

Juan M. Corchado Actualmente es Decano de la Facultad de Ciencias y Profesor Titular en el departamento de Informática y Automática en la Universidad de Salamanca (España). Obtuvo un doctorado en Ciencias de la Computación por la Universidad de Salamanca en 1998 y un doctorado en Inteligencia Artificial por la Universidad de Paisley (Glasgow, Reino Unido) en 2000. Es Director del grupo de Bioinformatica, Sistemas Inteligentes y Tecnología Educativa (http://bisite.usal.es) y Director del Máster en Comercio Electrónico de la Universidad de Salamanca (España). Anteriormente fue sub-director de la Escuela de Informática de la Universidad de Vigo (España) (1999-00) e investigador en la Universidad de Paisley (Reino Unido, 1995-98). Ha sido investigador colaborador en el Plymouth Marine Laboratory (Reino Unido) desde 1993. Ha liderado múltiples proyectos de investigación financiados por diversas instituciones españolas y europeas, públicas y privadas, y ha dirigido ocho tesis doctorales. Es co-autor de aproximadamente 150 libros, capítulos de libros, artículos en revistas internacionales, etc. publicados por organizaciones tales como Elsevier, IEEE, IEE, ACM, AAAI, Springer Verlag, Morgan Kaufmann, etc., y la mayor parte de ellos presentan investigaciones teóricas y prácticas en el campo de la Inteligencia Artificial y los sistemas distribuidos. Ha sido Presidente de numerosos comités organizadores y científicos en distintos simposios. 